\title{
Parasciences et procédés de légitimation
}

In: Revue française de sociologie. 1986, 27-2. pp. 205-219. 


\section{Résumé}

La notion de parascience recouvre un ensemble composite de pratiques et de doctrines rejetées par les confessions dominantes et la science officielle. Intimement apparentées à l'occultisme par leurs fonctionnements logiques et leur vision du monde, elles ne s'en distinguent que par la revendication d'un statut de science et la recherche d'une légitimité par la science. L'analyse des revues et ouvrages spécialisés, des procédés promotionnels et des formes de transmission du savoir fait apparaître un décalage entre une disposition à reconnaître les signes de légitimité culturelle et la capacité à maîtriser ces signes. Les erreurs engendrées par cet écart accréditent l'hypothèse d'une domination symbolique du savoir dominant s'exerçant sur les professionnels des parasciences et corrélative d'une attitude ambivalente. La science et son exercice fonctionnent à la fois comme vecteurs d'une domination subie et comme un système de signes réinterprétés alimentant une contestation de la hiérarchie des valeurs culturelles et une mise en question des principes de classement social.

\section{Resumen}

Gérard Chevalier : Paraciencias y modos de legitimación.

La noción de paraciencia recubre un conjunto compuesto de prácticas y de doctrinas rechazadas por las confesiones dominantes y la ciencia oficial. Intimamente aparentadas al ocultismo por sus funcionamientos lógicos y su vision del mundo, ellas no se distinguen de ello mas que por la revindicación de un estatuto de ciencia y la busqueda de una legitimidad por la ciencia. El análisis de las revistas y obras especializadas, de los modos promocionales y de las formas de transmisión del saber pone de manifiesto una diferencia entre una disposition de reconcer los signos de legitimidad cultural y la capacidad de dominar aquellos signos. Los errores engendrados por esta diferencia autorizan la hipótesis de una domination simbólica del saber dominante manifestandose en los profesionales de las paraciencias y correlativa de una actitud ambivalente. La ciencia y su desempeño funcionan a la vez como vectores de una dominación sufrida y como un sistema de signos reinterpretados, alimentando una contestación de la jerarquia de valores culturales y una puesta en duda de los principios de clasificación social.

\section{Zusammenfassung}

Gérard Chevalier : Parawissenschaften und Legitimierungsverfahren.

Der Begriff der Parawissenschaften umfasst eine zusammengewurfelte Zahl von Praktiken und Doktrinen, die von den herrschenden Konfessionen und der offiziellen Wissenschaft verworfen werden. Sie sind eng mit dem Okkultismus durch ihre logische Arbeitsweise und ihre Weltanschauung verwandt und unterscheiden sich von ihm lediglich durch ihre Forderung nach einem Wissenschaftsstatus und das Streben nach der Legitimierung durch die Wissenschaft. Die Untersuchung der spezialisierten Zeitschriften und Werke, der Promotionsverfahren und der Art der Wissensvermittlung zeigt eine Verschiebung zwischen einer Bereitschaft, die Zeichen der kulturellen Legitimität anzuerkennen und die Fähigkeit diese Zeichen zu meistern. Die durch diese Spaltung entstehenden Irrtümer begründen die Hypothese einer symbolischen Beherrschung des herrschenden Wissens auf die beruflichen Ausuber der Parawissenschaften, Beherrschung, die aus einer zwiespältigen Haltung entsteht. Die Wissenschaft und ihre Ausubung wirken sowohl als Vektoren einer erlittenen Beherrschung als auch als System von neu interpretierten Zeichen, die Stoff zur Anfechtung der Hierarchie der kulturellen Werte liefert und zur Infragestellung der Grundsätze der sozialen Einstufung.

\section{Abstract}

Gérard Chevalier : Parasciences and legitimizing procedures.

The notion of parascience covers a mixed grouping of practices and doctrines rejected by main religions and official science. Intimately linked to occultism in their logical functioning and view of the world, they are distinguished only by their demand to be recognized and legitimated as a research science by the scientific community. The analysis of journals and specialized works, promotional proceedings and ways of transmitting knowledge shows a gap between a readiness to acknowledge the signs of cultural 
legitimacy and the ability to master these same signs. The mistakes generated by this discrepancy add credibility to the hypothesis of a symbolic domination of prevailing knowledge which weighs on professionals of the parasciences and accompanies an ambivalent attitude. Science and the practice thereof operates at the same time as a carrier of an experienced domination and as a system of reinterpreted signs adding fuel to a fire of dispute over the questioning of the hierarchy of cultural values and the principles of social classification. 


\title{
Gérard CHEVALIER
}

\section{Parasciences et procédés de légitimation}

\begin{abstract}
RESUME
La notion de parascience recouvre un ensemble composite de pratiques et de doctrines rejetées par les confessions dominantes et la science officielle. Intimement apparentées à l'occultisme par leurs fonctionnements logiques et leur vision du monde, elles ne s'en distinguent que par la revendication d'un statut de science et la recherche d'une légitimité par la science. L'analyse des revues et ouvrages spécialisés, des procédés promotionnels et des formes de transmission du savoir fait apparaître un décalage entre une disposition à reconnaître les signes de légitimité culturelle et la capacité à maîtriser ces signes. Les erreurs engendrées par cet écart accréditent l'hypothèse d'une domination symbolique du savoir dominant s'exerçant sur les professionnels des parasciences et corrélative d'une attitude ambivalente. La science et son exercice fonctionnent à la fois comme vecteurs d'une domination subie et comme un système de signes réinterprétés alimentant une contestation de la hiérarchie des valeurs culturelles et une mise en question des principes de classement social.
\end{abstract}

Astrologie, parapsychologie, radiesthésie, géobiologie, la notion de parascience est un terme commode pour désigner un ensemble composite de pratiques et de doctrines rejetées par les confessions dominantes et la science officielle. Néologisme instable qui voudrait faire oublier les limites institutionnelles de la pratique scientifique, elle évoque une zone imprécise entre savoir et croyance. Circonscrire cette nébuleuse suppose d'abandonner la catégorie-écran de parascience et de revenir à la notion sous-jacente d'occultisme. L'ensemble des démarches ordinairement rangées sous cette rubrique fournit en effet une base de compréhension plus solide. Que l'on considère les méthodes divinatoires traditionnelles (e.g. chiromancie, tarots, numérologie) ou les diverses formes d'astrologie, on retrouve la même vision du monde unifiée par la théorie des correspondances (1). Par ailleurs, la transposition à l'occultisme des résultats apportés par l'analyse phénoménologique des mythologies primitives (2) fait apparaître des fonctionnements intellectuels identiques, et constants pour l'ensemble des

(1) Cette théorie, formulée au Xville siècle par le Suédois Swedenborg, peut être considérée comme le soubassement de tout l'occultisme. Elle pose que "tout objet appartient à un ensemble unique et possède avec tout autre élément de cet ensemble des rapports nécessaires, intentionnels, non temporels et non spatiaux ». Cf. R. Amadou. Loccultisme. esquisse d'un monde virant, Paris, Ed. Julliard, 1950 et Le Monde incommu, n" 45.

(2) Cf. E. Cassirer, La philosophie des formes simboliques, tome II: La pensée mythique, Paris, Ed. de Minuit, 1972. 
doctrines considérées (3). Si l'on analyse enfin les théorisations courantes de la parapsychologie, de la radiesthésie, de la biorythmie, de la géobiologie, de la biodynamie, etc., on constate que les processus logiques sont les mêmes qu'au sein de l'occultisme et que la vision du monde qu'elles actualisent lui est intimement apparentée (4).

Ces premiers repères posés, certains critères de définition peuvent être avancés. Apparaissent comme parasciences toutes les doctrines liées à l'occultisme (5), rejetées par les sciences officielles mais revendiquant le statut de science ou recherchant une légitimité par la science. Cependant, cette délimitation de l'objet ne dissipe pas son opacité générique. Sauf à imaginer un dispositif de recherche capable d'appréhender tous les aspects de cette réalité polymorphe, il n'est pas d'autre issue que de la découper en segments. Deux cas de figure se présentent généralement : soit le découpage se réfère à une théorie de l'objet établie par d'autres travaux, soit il s'appuie sur un repérage préliminaire des formes élémentaires de son existence sociale. Parmi celles-ci, les modes de commercialisation représentent une dimension essentielle. Les "journées", salons, collections et revues spécialisées, les écoles et les stages de formation, enfin les " consultations" et plus largement les prestations de services constituent les signes concrets de l'existence d'un marché, donc de publics, de clientèles et de professionnels.

En relation à la définition esquissée plus haut, dans laquelle la science apparaît comme le pôle magnétique par rapport auquel s'orientent toutes ces démarches, il est apparu important d'analyser les procédés employés par ces professionnels pour pallier l'exclusion de leurs disciplines de la sphère des connaissances institutionnellement garanties et pour s'approprier une part de la légitimité de celles-là.

L'étude de cet objet partiel présente plusieurs avantages pour la compréhension des parasciences. Elle permet, en premier lieu, d'ouvrir une autre perspective que celle tracée par la compréhension spontanée en

(3) Cf. G. Chevalier, Pensée mythique et rapport au savoir. Contribution à une sociologie des nouvelles formes de croyances, thèse de $3^{\text {e }}$ cycle Paris VIII, 1983, I, chap. III.

(4) Les concepts scientifiques, saisis dans leur modalité mythique, forment avec les notions occultistes traditionnelles un registre étendu, dont la fonction logique est de servir de médiation entre tous les éléments perceptibles ou imperceptibles de l'environnement. Les relations symboliques, analogiques, l'astral, l'éther, l'énergie, les vibrations, les ondes, les rayons, les rayonnements, les radiations, les champs (bio-énergétiques, bio-plasmiques, bio-gravitationnels, bio-électriques), le fluide, tous ces termes sont l'expression d'une même idée, au fondement de loccultisme comme des parasciences, celle de la participation dans l'invisible, de la diversité infinie du perçu à l'unité structurelle de la totalité.

(5) A l'homologie des fonctionnements logiques s'ajoute une homologie fonctionnelle attestée par la présence régulière des parasciences aux côtés des formes traditionnelles d'occultisme. Que l'on flâne devant la vitrine d'une librairie spécialisée, qu'on lise des revues consacrées à l'« étrange " (e.g. L'Inconnu, L'Autre Monde, Nostra, L'Inexplique) ou qu'on visite une des manifestations commerciales apparues au début des années 80 (e.g. Journées Coresprit, Salons Majolaine, Forums Européens, Bio/Psy), on retrouve systématiquement l'alliance obligée, bien que variable dans ses proportions, des démarches évoquées précédemment. 
termes de croyances. Les multiples emprunts aux disciplines scientifiques, qui émaillent la littérature parascientifique ou encore les stratégies de légitimation adoptées par les astrologues (e.g. utilisation d'un ordinateur), indiquent que ces croyances se donnent et vraisemblablement fonctionnent comme des savoirs. Corrélativement, se pose la question de la place occupée par les tenants du paranormal dans la distribution des instruments d'appropriation culturelle et de leur attitude envers le savoir légitime. A cet égard, l'analyse des revues et ouvrages spécialisés, des procédés promotionnels et des formes de transmission du savoir fait apparaître un écart entre connaissance et reconnaissance culturelle générateur d'erreurs récurrentes.

En second lieu, les exemples de ce décalage entre aspirations et compétences suggèrent des hypothèses ponctuelles sur les modalités de réception au sein du public. Il ne s'agit pas de faire dire au matériel étudié plus qu'il ne le peut. Cependant, le rapport ambivalent et autodidacte à la science officielle qui marque les productions parascientifiques n'aurait vraisemblablement qu'un faible rendement commercial si aucune disposition convergente n'existait au sein du public. Les représentations de la compétence et de la posture légitime ne peuvent se comprendre indépendamment d'un accord sur le sens des signes, y compris dans l'erreur, entre les tenants des parasciences, leurs lecteurs ou leurs clients (6).

(6) L'ensemble de l'analyse se fonde principalement sur un corpus de 19 ouvrages spécialisés : soit 4 ouvrages sur la parapsychologie, I sur les ovNi. I sur la géobiologie. 3 sur l'archéologie-fiction, 1 sur la biorythmie, I sur la radiesthésie, I sur la nouvelle gnose, 2 sur la théosophie, 1 sur la biodynamie, 2 sur l'astrologie, 1 sur l'occultisme en général et l sur les "grandes énigmes de l'univers ». Revues: L'Autre Monde, $\mathrm{n}^{\circ} 8,9$, 45, 46, 47, 51, 52, 54; L'Inexpliqué, $\mathrm{n}^{\text {os }} 1,2$, 3: Le Monde inconmu, $\mathrm{n}^{\text {os }} 12$ et 14; Nostra. $\mathrm{n}^{\text {is }} 264$ et 505; L'Incommu, nos 16 et 65 ; Gnose, $\mathrm{n}^{\circ}$ 1; Ere Nouvelle, $\mathrm{n}^{\circ} \mathrm{I}$; Astrologique, $\mathrm{n}^{\circ} 10$; Le Narurel, $\mathrm{n}^{\circ}$ 3; Carré, $\mathrm{n}^{\text {os }}$ 10-11; Grande Conjonction, $\mathrm{n}^{\text {os }} 3$ et 4 ; Astral, $\mathrm{n}^{\text {os }} 278$ à 287

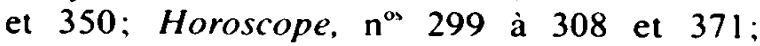
Astres, $\mathrm{n}^{\text {os }} 322$ à 331 et 251: Eıdudes Abelliennes, $\mathrm{n}^{\circ} 1$; Parapsychologie, nos 10 et 11.

Ces ouvrages et revues ont été choisis dans le but de couvrir lensemble du syncrétisme étudié. Quand les éléments de compréhension apportés par les ouvrages se sont révélés insuffisants, on s'est appuyé sur ceux fournis par la documentation recueillie. Ne s’agissant que de rendre compte d'un ensemble cohérent de thèmes, de démarches intellectuelles et de procédés de production de la légitimité, lanalyse de contenu classique, centrée sur l'étude des significations et des implications logiques, ne nécessitait pas expressément le renfort d’approches procédant par découpage d'unités sémantiques, repérage des relations syntaxiques et quantification. La recherche des déterminants sociaux conditionnant le cheminement du discours, en particulier l'ascendant exercé par les modèles discursifs savants, imposa cependant à la lecture de ne pas s'en tenir exclusivement au contenu manifeste et de sorienter vers les contradictions que les caractéristiques sociales et les convictions des auteurs engendrent dans l'écriture. On fut amené ainsi à qualifier le contenu en fonction des représentations de la posture légitime $\mathrm{du}$ locuteur, de la démonstration, des modalités d'intégration des éléments de connaissance et à rechercher les incohérences entre ces différents plans. Par ailleurs, la mise en évidence d'un écart entre revendications culturelles et compétences, non plus au niveau latent d'une signification sociale dissimulée par le sens littéral des mots, mais au niveau manifeste de l'utilisation des savoirs homologués, dut s'armer de considérations biologiques, physiques ou physiologiques généralement étrangères aux travaux sociologiques, mais imposées par la nécessité de tracer les limites des connaissances investies. 


\section{I. - La célébration des sciences}

La forme du savoir légitime revendiquée en priorité par les parasciences est indiscutablement la science. Qu'elle soit "humaine ", physique ou naturelle, elle s'impose comme l'ensemble des critères de certification du vrai, institutionnellement garantis et repris en écho par toutes les initiatives qui, dans le champ de production symbolique, visent à en tirer profit. Le bénéfice de nombreux vecteurs de diffusion n'empêche cependant pas que sa réalité objective échappe à tous ceux qui sont exclus de la participation concrète à sa production ou à sa reproduction institutionnelles. La vulgarisation scientifique, en tant qu'ensemble des circuits parallèles à l'institution scolaire, par lesquels des résultats expérimentaux se font connaître, change en vérités "absolues" des acquis provisoires qui ne prennent leur sens réel que par la pratique de recherche. Les profanes n'ayant pas l'expérience critique de la pratique scientifique ne peuvent que s'entendre raconter la science (7). En ce sens, la science objective se transforme en représentations sociales, selon des modalités de restructuration qui varient en fonction des niveaux de formation scientifique et de la place tenue par ce type de savoir dans le capital culturel d'une classe d'agents. L'usage de connaissances scientifiques dans les parasciences représente, en quelque sorte, un degré supplémentaire dans la promotion ontologique de résultats et de démarches expérimentales : des représentations socialement constituées sont redéfinies par leur intégration aux disciplines mythiques et systématiquement exhibées comme références indubitables. La science de l'occultisme est une science reconstruite par des profanes à partir de la vulgarisation et retransmise à d'autres profanes à l'appui d'une vision particulière du monde. La distance à la pratique légitime s'en trouve multipliée par deux, sinon par trois, à la réception finale. Naturellement, toutes les utilisations de sciences patentées observables dans notre corpus ne sont pas appréciables d'une manière uniforme. Ouvrages et revues reflètent des niveaux de compétence variables, dont l'évaluation échappe à l'investigation proprement sociologique. Pourtant la domination symbolique du savoir légitime n'en est pas moins manifestée par la multiplicité des références mises en œuvre et par la place qui leur est faite dans la syntaxe. La domination du savoir institutionnellement garanti est si puissante que la perception de l'usage légitime des références peut prendre, dans les versions commerciales de la "pensée traditionnelle " (8), l'allure d'une profusion insensée. Un article paru dans la revue Le Monde inconnu(9), visant à démontrer, si toutefois on en a saisi le

(7) Cf. P. Roqueplo, Le partage du savoir, Paris, Ed. du Seuil, 1974.

(8) On entend par cette expression l'ensemble des démarches qui, à travers l'étude de l'hermétisme, du symbolisme, de la Kabbale, visent à retrouver "une tradition pri- mordiale " révélée à l'aube de l'humanité et vers laquelle convergeraient tous les cultes esotériques.

(9) S. Bénatouil, «Intuition et révélation ", Le Monde inconnu, $\mathrm{n}^{\circ}$ 14, janvier 1981, pp. 25-27. 
véritable sens, que le "génie humain " n'a de raison d'exister que s'il accède à la vérité divine, constitue une bonne illustration de cette angoisse de la certification, en même temps que du renforcement des limites de la compétence scolaire par la croyance.

Dès les premières phrases, le lecteur peut éprouver la sensation rassurante d'être associé à la recherche ardue d'une autorité confirmée : "Intuition et révélation, mais comment ? Par les vibrations, les ondes. Mais avec qui ou avec quoi ? Voilà ce qu'il nous faut découvrir ". Plus loin: "Parmi les particules et ondes, celles de la sexualité sublime, insoupçonnable (sic). Nous allons en découvrir l'existence ». Dans le même temps, les références invraisemblables à la physique se succèdent. A propos des ondes, l'auteur note : «Depuis celles formées par la lumière sur une tache d'huile, d'autres sur le bitume, les ondes sonores radio (alors qu'il s'agit d'ondes électromagnétiques), il y a celles nettement plus fines - ultrasons et autres - qu'aucun appareil ne peut capter ni émettre et qui sont les ondes mystérieuses qui suscitent les premières plus grossières ". Le seul point commun entre les ondes acoustiques et le spectre électromagnétique est leur nature ondulatoire; par ailleurs, on peut acheter un sifflet à ultrasons dans tous les magasins d'articles canins. Mais là ne s'arrête pas le développement d'une vision enchantée de l'univers et du "génie humain ». On apprend immédiatement à la suite que "des particules fantômes, invisibles, voire inexistantes, donc purement spirituelles, sont néanmoins présentes; elles parcourent et animent toute l'humanité, elles sont comme l'Esprit des choses ». Une version puérile de la physique quantique, dans laquelle on retrouve la modalité particulière de l'essence propre à la pensée mythique, vient ici à l'appui d'une vision magicopanthéiste du monde (10). Le rapport défavorisé à la culture scientifique se dégage avec une netteté particulière de la suite du propos. C'est une véritable réitération imaginaire de l'expérience scolaire qui se joue dans les deux pages suivantes. Par petits paragraphes successifs, l'auteur nous raconte la marche invincible de la Science vers le progrès spirituel de l'humanité. Depuis Newton jusqu'à Jean Rostand, en passant par Joseph Fraunhofer, Gustav Kirchoff, Bunsen, John Herschell, la découverte du radiotélécospe, le prix Nobel de physique Jean Perrin, Pavlov et Lavoisier, se dessine une quasi-relation pédagogique, dans laquelle la diffusion de thèmes mythiques s'identifie à l'appropriation défavorisée de connaissances scolaires. Le privilège de tirer "un bilan", qui " met en évidence l'importance et l'audace théorique de diverses recherches en cours",

(10) Le procédé d’ancrage de la pensée mythique à la science de pointe se retrouve dans la majorité des spéculations du courant sur-rationaliste. Il fit les beaux jours du Matin des magiciens ainsi que de la revue Planète et réapparaît intact, comme le note M. Rouzé, dans la parapsychologie savante actuelle. "Il s'agit d’expliquer les 'phénomè- nes paranormaux, en s'appuyant sur des interprétations extrêmes de la physique quantique et de valoriser, par opposition à la connaissance scientifique telle qu'elle s'est édifiée en Occident depuis la Renaissance, les approches mystiques de lunivers et les philosophies orientales ". M. Rouzé, "Liimposture de Cordoue ". Science et vie, n" 760 
contribue à créer l'illusion d'une position dans le champ scientifique propice à l'information immédiate sur l'état des travaux et à son évaluation qualifiée. On peut cependant gager que de tels artifices passent inaperçus pour un public dont les intérêts culturels et la vision du monde s'ajustent à ceux de l'auteur. Que les connaissances investies soient vraies ou fausses n'a finalement pas grande importance, pourvu que les signes de la légitimité culturelle déployés puissent être reconnus comme authentiques et le discours perçu à travers les filtres superposés de la reconnaissance/méconnaissance culturelle et de la croyance mythique.

Toutefois, l'articulation des connaissances scientifiques et des conceptions parascientifiques peut engendrer certaines difficultés, dont l'ignorance témoigne d'une représentation dominée de la démarche scientifique. L'intégration du déterminisme astrologique au détail du système nerveux pose, par exemple, quelques problèmes de médiation. L'astrologie médicale fournit de nombreux exemples de cette rupture entre une volonté d'exactitude scientifique et les implications de la vision cosmologique qui la fonde : "C'est un signe de type émotif (le Lion) et, comme le Bélier et le Sagittaire, un signe de feu, donc un signe d'Energie Vitale. Le signe du Lion est régi par : la colonne vertébrale, la moëlle épinière, le cœur, le pancréas. La moëlle épinière repose dans le canal rachidien. Elle est formée de deux substances. Au centre, de la substance grise contient des cellules motrices multipolaires. Leurs "neurites" forment les fibres des racines antérieures ou motrices des nerfs spinaux. La corne postérieure se compose de cellules sensibles (...). La corne latérale forme un territoire étroit dont les groupes cellulaires appartiennent au système nerveux autonome. Ce sont surtout les cellules ganglionnaires sympathiques dans la partie lombaire et thoracique (...). En résumé, pour la substance grise, les cellules des cornes postérieures sont des relais pour les conducteurs sensibles (...). Quant à la substance blanche, sa fonction est une conduction des influx moteurs et sensibles tant à l'intérieur de la moëlle épinière que vers les centres supérieurs (cervelet et thalamus). C'est pourquoi le Lion ne peut être qu'un signe émotif, puisque tout ce circuit est le centre des sensations " (11).

Ce luxe de détails, dont nous avons laissé de côté certains points pour ne pas alourdir une citation déjà longue, ne prend son sens littéral que par référence à une représentation particulière de la démonstration. Il n'apporte strictement rien à l'appui du déterminisme astrologique, puisque la description neurologique se déploie pour elle-même. L'ensemble revient à dire textuellement : un Lion est régi par la moëlle épinière dont voici la description, or la moëlle épinière est le lieu de l'émotivité (" puisque... sensations "), donc le Lion est un émotif. En rétablissant l'ordre du syllogisme, on distingue plus facilement la fonction réelle de la référence scientifique. Ce qui pourrait constituer la proposition mineure (« puisque...

(11) J. Mares, « Astrologie médicale : le Lion", L'Autre Monde, n 52, p. 52. 
sensations ") réalise l'amalgame implicite entre les sensations, c'est-à-dire la réception sensorielle généralement indissociable d'une structure perceptive complexe, et l'émotivité. C'est une question que l'auteur règle discrètement en cinq mots, bien qu'elle constitue l'articulation principale de son raisonnement, précisément parce que cette implication est totalement arbitraire et que son encyclopédie médicale ne peut y apporter aucune réponse. Mais la majeure est plus significative encore; l'organisation d'une représentation de la légitimité y procède par déplacement de la signification. Alors que ce qu'il importe logiquement d'étayer est précisément que les natifs du Lion sont "régis", entre autres, par la moëlle épinière, l'astrologue concentre son effort de compilation sur ce qui s'avère le plus propice à produire l'impression de la légitimité culturelle, en même temps que le moins utile à la démonstration : la description du système nerveux central. L'absence d'intégration logique de la description neurologique rend d'autant plus tangible la valeur de l'enjeu qu'un savoir certifié représente pour les tenants des parasciences. La domination symbolique dont ils sont victimes, quand elle ne se lit pas au plan du savoir, se lit au plan de son utilisation.

\section{II. - La fascination universitaire}

Le recours aux hautes autorités universitaires et académiques est une constante des procédés promotionnels de la culture moyenne. Les parasciences en appellent ainsi à un nombre considérable de professeurs et de docteurs en tous genres. En 1976-77, durant les grandes offensives commerciales que suscita la parapsychologie, l'éditeur $R$. Laffont ne craignait pas d'user largement de l'effet de certification des grades universitaires, quitte à faire parler les morts. Un dépliant publicitaire grand format qui nous fut adressé en 1977 présente ainsi les quatorze volumes de la collection La parapsychologie ou les pouvoirs inconnus de l'homme (12): "Une pléiade de grands écrivains et de professeurs ont collaboré à ces 14 volumes passionnants ". Suit l'énumération verticale des quatorze titres et des thèmes développés, puis, dans un encadré orangé : "Voici les noms des auteurs qui ont collaboré à notre collection", "Les citer tous serait impossible dans le cadre de ce message. Mais vous lirez entre autres des textes de ... ". Parmi les vingt-deux professeurs et docteurs cités, ont été glissés les tenants de la métapsychique du siècle dernier, qui n'ont pu de toute évidence être contactés que par l'intermédiaire d'un médium ou du guéridon traditionnel : Pr. H. Bernheim (mort en 1919), Pr. W. Crookes (mort en 1919), Pr. G. Geley (mort en 1924), Dr. E. Osty (mort en 1938), Pr. Ch. Richet (mort en 1935). Même s'il est probable que la

(12) La souscription à la collection donnait également droit à deux cadeaux : un

pendule doré et son étui. 


\section{Revue française de sociologie}

datation correcte des citations soit rétablie dans les ouvrages, l'exploitation du pouvoir symbolique accumulé par l'enseignement supérieur s'inscrit dans une stratégie de stimulation de la disposition à la reconnaissance culturelle, consciente dans ce cas, du niveau de méconnaissance du public visé, sinon du renforcement de celui-ci par l'exhibition de compétences d'élite. Le déploiement d'un écran de légitimité témoigne d'une inquiétude du statut qui hante les parasciences et dont les moyens concrets sont du même ordre que ceux énoncés aux pages précédentes. Chaque possibilité de mise en avant des titres scolaires est exploitée sans équivoque, parfois même chez des agents dont la position semblerait ne pas l'autoriser. Le professeur E.B. n'entend ainsi pas laisser passer la moindre occasion de faire état de ses "titres", sans peur des présentations ambiguës ou fallacieuses. Un encadré en pleine page du programme/guide pratique Coresprit 80 le présente en ces termes :

PROFESSEUR E.B.

Docteur ès-sciences - Docteur en bio-physique

SPÉCIALISÉ EN RADIESTHÉSIE -

PARAPSYCHOLOGIE - RADIO-PSYCHIE

CONSULTANT INTERNATIONAL en "Psycho-prospective "

DOCTEUR GRADUÉ • de l'Académie Nationale du Québec (Montréal)

- du North West London University

- de l'American Institute of science

- de l'Institut supérieur des sciences (Suisse)

PROFESSEUR A - l'Académie Nationale du Québec (Montréal)

- l'Université Populaire de Paris

- l’Université internationale - Aupac - Paris V

SECRÉTAIRE GÉNÉRAL de l'Institut Européen des Sciences Humaines

LAURÉAT de la Société Psychique Internationale

TITULAIRE des MÉDAILLES D'OR de l'INVENTION, salons internationaux de Bruxelles et Genève.

SÉMINAIRES D'ENSEIGNEMENT PRIVÉ

TITULAIRE de la carte INTER-PRESSE (I-723) pour ses articles de vulgarisation scientifique et ses chroniques de "Prospective financière et économique ".

ENSEIGNEMENT PRIVÉ : COURS, SÉMINAIRES 
L'accumulation des signes, vrais ou falsifiés, de la légitimité vise autant à accréditer la valeur de l'agent que celle du savoir hérétique dont il tire profit. L'hétérogénéité des preuves avancées (diplômes, fonctions diverses, prix, médailles, carte de presse) fait appel à une représentation de la légitimité culturelle comme collection volumineuse de distinctions démonstratives, plutôt que comme qualité intrinsèque d'une compétence certifiée.

Outre qu'on retrouve ici, nourrie d'une anxiété de la preuve (numéro de la carte de presse) en contradiction avec l'assurance et la retenue permises par la position revendiquée, la logique de l'accumulation comme démonstration de la valeur qui caractérise la littérature occultiste, les procédés de construction de cette image de marque se dessinent clairement dès qu'on $y$ porte attention. S'agit-il tout d'abord d'un ou de deux doctorats ? Il est d'usage de considérer qu'être docteur en bio-physique signifie être docteur ès sciences et la formulation correcte eût été : docteur ès sciences en bio-physique. Dans le cas inverse, il aurait été utile de préciser dans quelle spécialité distincte de la bio-physique a été effectué le premier doctorat. Mais il est probable que la multiplication du titre par lui-même vise à élever au carré son pouvoir de certification.

La réalité des compétences étrangères ne nous fut accessible que par la médiation des ambassades concernées. Les éléments recueillis ne sont cependant pas sans intérêt pour la compréhension de l'enjeu que constituent les certifications universitaires et les procédés employés pour les imiter. Les recherches entreprises à Montréal par les correspondants de la Délégation générale du Québec en France attestent que l'«Académie nationale du Québec " n'appartient ni à l'enseignement universitaire, ni à l'enseignement collégial canadien. Il s'agit selon toute vraisemblance d'une association privée, conçue sur le modèle des associations françaises citées plus bas. Notons également que le terme d'《académie " est réservé à l'enseignement secondaire au Québec et que cette province ne constitue pas à ce jour une entité nationale. La "North West London University » est un établissement d'enseignement privé dont les diplômes ne sont pas reconnus par l'Association des universités du Commonwealth, qui n'a pas reçu l'agrément royal et ne fait, à ce titre, pas partie de l'Université de Londres. D'après l'ouvrage édité chaque année par l'« American Council of Education " (13), il n'existe aucun établissement d'enseignement supérieur qui porte le nom d'" American Institute of Science ". Il en est de même en Suisse pour l'«Institut supérieur des sciences " puisque tous les instituts spécialisés se trouvent dans les facultés des sciences et que cette institution ne fait pas partie de l'université.

Ces indices rejoignent ceux apportés par les activités d'enseignement en France. L'Alliance universelle pour la paix et la connaissance (AUPAC) est une association à but non lucratif, organisant des conférences gratuites sur les traditions spirituelles et le paranormal. Renseignements pris auprès de l'administration de l'Université de Paris $v$, il n'existe aucun lien entre les

(13) Accredited institutions of post secondary education, 1982-1983. 
enseignements dispensés à la Faculté de médecine et les activités de l'AUPAC. Cette association n'a pour toute relation avec l'Université que la location d'un amphithéâtre un samedi sur deux, comme peut y prétendre n'importe quelle autre organisation (14). Naturellement, il est plus rentable de donner à croire qu'il s'agit d'un enseignement officiel.

Le même traitement est réservé à l'Université populaire de Paris, dans deux articles parus dans la revue L'Autre Monde (15). Il s'agit là d'une association sans agrément particulier, et dont les intervenants sont bénévoles. Parmi les domaines abordés : économie, sciences, techniques, arts, il en est de favorables à la transmission magistrale des thèmes mythiques. "La vérité voilée ", rubrique sous laquelle furent traités depuis la création de cette association des sujets comme "Le visage dans l'obscur ou le guide intérieur. Les rêves en tant que messages. L'espace du dedans. Les livres des Morts tibétain, égyptien et la science ", ou encore une rubrique "Santé morale - Equilibre social " sous laquelle sont classés des thèmes comme "Comprendre l'être humain dans sa globalité : corps énergétique, psychique, spirituel et ses rapports avec le monde vivant "; enfin, on retrouve au chapitre de la "Connaissance du monde et de soi " les variations classiques sur le tellurisme : "Mystère du sous-sol, ondes nocives, ondes bénéfiques, relations avec le cosmos ». Des cours sont également organisés (électronique, informatique, etc.) qui ne se relient pas à ces conceptions. De même que dans les manifestations commerciales apparues au début des années 80 (e.g. Salons Marjolaine), les thèmes mythiques apparaissent dans des proportions variables et tendent à se banaliser au contact des savoirs plus neutres. Le professeur E.B. recourt au même procédé à propos de cet organisme. Renvoyant à ses propres prestations, il note : "Histoire théorique et pratique de la radiesthésie informationnelle - Communication du professeur E.B. à L'Université populaire de Paris-Sorbonne Amphithéâtre Gaston Bachelard le 18 Octobre 1980 ». Dans l'article du mois suivant, il récidive : "Cours-entretiens qui sont extraits des communications et cours que j'ai développés à l'Académie nationale du Québec (Montréal), en Sorbonne, à l'Université populaire... ". Selon le même principe que précédemment, n'importe quelle association peut louer l'amphithéâtre Bachelard, ce dont profite également la Nouvelle Acropole (16). L'Université populaire recourt par ailleurs aux locaux de la Faculté de droit, à ceux de l'Ecole nationale des PTT et à ceux de trois centres socio-culturels.

Cette inquiétude de la légitimité, qui va jusqu'au truquage, impose bien entendu le choix des institutions les plus prestigieuses. Les exemples

(14) Le bénéfice tiré de ce lieu prestigieux se révèle d’autant mieux un enjeu crucial que le nombre de participants n'excède jamais une quarantaine de personnes, alors que l'amphithéâtre loué peut en contenir cent quatre-vingts.

(15) E. Baer, "Introduction à la radiesthésie informationnelle $", n^{\circ} 46$.
(16) L'association Nouvelle Acropole organise des conférences pluri-hebdomadaires sur des sujets tels que la cosmogonie des anciens Mayas, Pythagore et l'alchimie des nombres, la symbolique des labyrinthes, les grands sanctuaires initiatiques de la Grèce, etc. Cette organisation est implantée dans plusieurs pays européens. 
précédents sont à replacer dans la logique de la domination symbolique qu'exerce le champ intellectuel, et à l'intérieur de celui-ci les institutions universitaires, sur les parasciences. Les formulations ambiguës ou trompeuses ne tirent leur efficacité que de la bonne volonté culturelle désarmée de certaines fractions de classes.

Entre l'erreur consciente de ces procédés et l'erreur probablement inconsciente des références scientifiques citées plus haut, n'existe qu'une différence de compétence à maîtriser un même projet. Dans ce domaine, la marge est souvent faible entre le vrai, le vraisemblable et le faux. Seule importe l'organisation, de bonne ou de mauvaise foi, d'un spectacle de la légitimité qui dissuade la clientèle de chercher plus avant. Le plus souvent, les falsifications découvertes sont insignifiantes, un simple petit décalage, presque une faute de frappe, un trait d'union malencontreux; elles composent avec une part de vérité dont il s'agit de multiplier le pouvoir de certification.

\section{III. - Une inculcation quasi scolaire}

Parmi les indices qui témoignent de l'importance que revêtent, dans une fraction dominée du champ de production symbolique, les sanctions de l'enseignement supérieur, les formes de transmission du savoir sont certainement les plus significatives. La majorité des structures repérables revendique explicitement, par les noms qu'elle adopte, la participation à l'inculcation légitime d'un savoir savant : "Haute Ecole de Recherche " (Université AUPAC), "Université Internationale d'Astrologie " (en fait une simple boîte aux lettres), "Faculté de Parapsychologie de Paris ", "Académie de Parapsychologie ", "Université Verte » et, disparus ou rebaptisés depuis peu : l'" Ecole Supérieure d'Astrologie de Paris ", l'" Académie Internationale d'Astrologie ", le " Mouvement pour l'Astrologie à l'Université ", la « Faculté d'Astrologie ".

Ces appellations prises à leur valeur faciale dissimulent des entreprises diversifiées par leur degré de réussite et leur objet réel. Les "enseignements " parapsychologiques, par exemple, recouvrent aussi bien les procédés divinatoires traditionnels que l'étude du symbolisme ou les démonstrations de voyance. Derrière les "Universités ", se cachent autant des associations puissantes (Université Verte) que des agents relevant à intervalles réguliers le courrier d'une boîte aux lettres.

Parmi les diverses démarches relevant des parasciences, l'astrologie apparaît comme la plus propice à une reproduction scolaire. L'importance des références aux savoirs homologués, la forme et la durée des cycles d'enseignement, les buts pédagogiques revendiqués et le statut accordé au diplôme ou au certificat de fin d'études donnent la mesure de l'attraction exercée par l'appareil d'enseignement officiel. 


\section{Revue française de sociologie}

Les deux écoles d'astrologie visitées ont disparu en 1980 avec le départ en province de leurs animateurs. On en rapportera les aspects principaux, relativement à notre hypothèse. L'Ecole Supérieure d'Astrologie de Paris, dont l'appellation elle-même marquait d'autant plus nettement la revendication académique de son fondateur que n'existait pas d'établissement représentant le niveau moyen ou primaire, consistait en une petite salle d'environ trente places, soigneusement tapissée et donnant directement sur la rue. L'ensemble du programme ayant donné lieu à des enregistrements destinés à la vente par correspondance, le caractère magistral du cours auquel on a assisté dérivait sur le spectaculaire : plages de musique stéréophonique soulignant la grandeur des personnages historiques évoqués ou accentuant la profondeur des ténèbres de l'âme, synopsis d'ensemble parfaitement au point et intervenants bien entraînés. Les élèves, neuf femmes et quatre hommes, écoutaient religieusement comment La Brinvilliers et La Voisin ourdirent l'Affaire des Poisons ou pourquoi Madame Bovary et Thérèse Desqueyroux symbolisent le "poison littéraire ". Ils ne furent à aucun moment invités à s'exprimer. Le contenu du cours lui-même comprenait un nombre important d'illustrations historiques qu'encadraient quelques « spots » astro-psychanalytiques, marquant principalement les têtes de chapitres et les articulations.

La lecture du programme complet de l'année 1976-77 apporte d'autres éléments. L'enseignement était divisé en trois degrés répartis sur trois ans. A raison de trente et un cours par an, étaient successivement abordés les trois degrés suivants :

- les bases de l'astrologie traditionnelle, illustrées par les thèmes de personnages célèbres;

- une méthode d'interprétation révolutionnaire : l'astro-psychanalyse, cycle dans lequel était faite une large place à la psychanalyse, sous une forme fortement dramatisée et métaphorique, par exemple : " La lune et son rôle dans la centrale psychique du ça : le royaume de la nuit. La jungle des instincts. Le monde des rêves. Le déferlement de l'émotion ", ou encore, comme titre d'une séance : "Les sourds dégâts du refoulement, le lac noir des névroses; du refoulement à la perversion, quand Satan conduit le bal »;

- enfin le degré terminal consacré à la mythologie et à l'histoire de l'astrologie.

A la fin du premier et du second degré, les élèves ayant suivi l'intégralité des cours étaient autorisés à se présenter à un examen de passage leur conférant, s'ils obtenaient la moyenne, les certificats A ou B. Les corrigés types fournis avec le programme révèlent les difficultés d'intégration d'un savoir systématique, comme la psychanalyse, aux correspondances astromythologiques. La psychanalyse manifestait d'autant mieux sa fonction de légitimation que ne lui était concédée dans les questions qu'une place secondaire, en contradiction avec l'orientation proclamée de la méthode et les intitulés de cours. Il s'agissait moins d'une «astro-psychanalyse » 
que d'une astrologie nourrie de mythologie grecque. L'examen du second niveau comprenait par exemple trois parties : le calcul et le montage d'une carte céleste, la réponse à six questions et l'interprétation d'un thème astrologique. Parmi les six questions, comprenant elles-mêmes cinq sous-questions, la référence à la psychanalyse n'apparaît au maximum qu'une fois par question, sous des formes se rattachant parfois au test psychologique dit "de complément de phrases" ou encore aux listes de questions à réponses rapides, utilisées dans les examens américains : " Freud avait envisagé le rêve sous deux aspects : le contenu manifeste et le contenu latent. Qu'est-ce que le contenu manifeste ? ", "Complétez cette phrase qu'il a prononcée par le mot qui manque : 'Le rêve est la voie royale vers...' "(17); "Comment se manifeste un complexe d'Orphée au niveau du psychisme d'un sujet marqué par ce complexe? ", réponse : "Au niveau du psychisme, le complexe d'Orphée prédispose l'être à un état de crise et d'angoisse. La vie prend, par moments, tournure de descente aux Enfers. L'être s'enferme en la ténèbre (sic) pour coïncider au destin et, s'il a la force créative, il remonte avec le pur joyau de son œuvre " (17). L'évitement de la dissertation, que des questions de cours censées appeler des réponses univoques imposaient durant les deux premières années, aboutissait paradoxalement à l'adoption d'une modalité de contrôle final fondée sur cette compétence scolaire. Les élèves ayant obtenu les certificats $A$ et $B$ et ayant suivi (ou acheté) l'intégralité des cours de troisième année étaient invités à rédiger et à soutenir durant trois heures une "thèse ", de cent à cent cinquante pages, leur conférant le titre de "Maître ès Sciences Astrologiques ". L'adoption mimétique des signes de l'inculcation légitime s'inscrivait dans un projet d'inspiration universitaire. La reprise du protocole de la soutenance de thèse et l'intitulé valorisant du diplôme, la rigidité d'un cursus arbitraire et la présence d'épreuves de barrage en contradiction avec l'impératif économique du nombre maximal d'élèves donnent la mesure de l'attraction exercée par l'Institution officielle.

La référence à la psychanalyse n'apportait par ailleurs qu'une légitimité de façade, les titres spectaculaires de telle ou telle séance se réduisant à quelques questions secondaires dans les épreuves de contrôle. En outre, celles-ci retrouvaient naturellement, comme on l'a vu, des formes en vigueur dans le premier cycle de l'enseignement secondaire, tout en préparant à la rédaction d'une " thèse ", elle-même conférant contre toute attente le titre de "maître ". On retrouve là le même écart entre reconnaissance et connaissance à l'origine de toutes les erreurs d'évaluation par lesquelles se révèle la domination dont l'occultisme est l'objet.

La seconde école visitée, l'Académie Internationale d'Astrologie, pratiquait la même reprise des apparences de l'action pédagogique légitime, sans toutefois que s'y manifestent des ambitions universitaires aussi nettes. Au terme d'un cursus sur quatre ans, les élèves obtenaient un diplôme privé sur lequel on a peu de renseignements. Le déroulement du cours

(17) Corrigé type de l'examen de fin de deuxième année. 


\section{Revue française de sociologie}

lui-même évoquait sans ambiguïté une classe du secondaire : diction proche de la dictée, exercices à faire pour la semaine suivante, interrogation terroriste des élèves, tous adultes, ayant des difficultés à manier les correspondances caractérologiques et, corrélativement, satisfecits décernés aux bons éléments. La réussite d'une telle formule auprès d'un public adulte, que l'animateur nous décrivit de la façon suivante : "Un chercheur en chimie (femme), un professeur d'anglais, un licencié en psychologie, une secrétaire de direction, une esthéticienne, une ex-comédienne sans profession, deux astrologues professionnels, un employé des PTT, un cadre moyen et quatre retraités ", nous semble devoir être référée aux effets cumulés des "filtres" évoqués plus haut (cf. supra p. 210).

La conjugaison de la croyance et de la reconnaissance/méconnaissance culturelle rejette dans l'impensé toute interrogation sur le sens de la pratique. L'adhésion à la représentation astrologique du monde neutralisant toute question sur l'arbitraire du mode d'imposition, la reproduction de la posture scolaire empêche la mise en question du contenu et réciproquement. La question de l'autorité pédagogique, que la croyance désamorce, paraît s'évanouir et même perdre toute chance de se poser, dès que sont reproduits les signes de l'imposition scolaire vécus durant un temps plus ou moins long par les participants. Il est par ailleurs vraisemblable que la légitimité de l'enseignant, indépendamment des certifications extérieures à l'astrologie (diplômes, qualification professionnelle), trouve dans l'emploi des données astronomiques et dans les calculs astrologiques l'appui d'un savoir constitué qu'on ne peut prétendre pratiquer sans l'avoir appris.

Les parasciences ne réalisent pas seulement l'intégration de bribes de savoir scientifique ou l'imitation des formes homologuées de son exercice. Elles se constituent en tant que fait social par et dans une relation spécifique au savoir institutionnellement garanti. Synthèses ambiguës d'une vision du monde et d'un rapport au savoir, ces "croyances " ne peuvent être envisagées indépendamment des formes sociales qui leur confèrent une originalité historique. La croyance en la science comme critère universel du vrai apparaît au moins aussi agissante, dans leur développement ou leur régression, que les croyances mythiques. Faire la sociologie des parasciences revient pour une part à analyser le fonctionnement social des sciences.

Toutefois la science officielle n'est pas simplement imitée. Elle est également mise en question. Les parasciences illustrent ainsi ce que J.-C. Passeron nomme "l'ambivalence significative" des cultures dominées (18). Le savoir légitime, son usage et son langage fonctionnent à la fois

(18) Cf. C. Grignon et J.-C. Passeron,. du CERCOM, n ${ }^{\circ}$, avril 1985. "A propos des cultures populaires ", Cahiers 
comme les instruments d'une domination subie et comme armes de résistance à la domination; intimement alliés à une vision mythique du monde, ils engendrent des formes culturelles dominées (les " para »-sciences), mais objectivement concurrentes des disciplines consacrées, comme l'atteste par exemple le rôle de psychologie de substitution que joue la psycho-astrologie dans le sens commun. La science et son exercice, en générant le tropisme parascientifique, apparaissent comme les vecteurs privilégiés d'une domination symbolique, mais également comme un système de signes réinterprétés alimentant une contestation de la hiérarchie des valeurs culturelles et une mise en question des principes de classement social. Si les parapsychologues jouent aux scientifiques, tout comme les guérisseurs aux médecins et les astrologues aux psychanalystes, c'est non seulement pour tirer le bénéfice de l'imitation du savoir légitime et de son exercice, mais aussi dans le but diffus et nécessaire pour certains groupes sociaux de transmuer progressivement en professions organisées des activités de substitution (19). Affirmer qu'une formation de "relaxologue " "donne accès à une profession de standing, épanouissante et particulièrement gratifiante au plan humain " (20) opère une sorte de bluff social dont l'usurpation des apparences de la légitimité culturelle constitue le moyen privilégié.

Gérard CHEVALIER CESDIP, CNRS 4. nue de Mondovi, 7500I Paris

(19) Cf. P. Bourdieu, La distinction. Critique sociale du jugement, Paris, Ed. de Minuit, 1979, pp. $427-428$.
(20) Documentation de l'Institut des Sagesses et Techniques Orientales. 